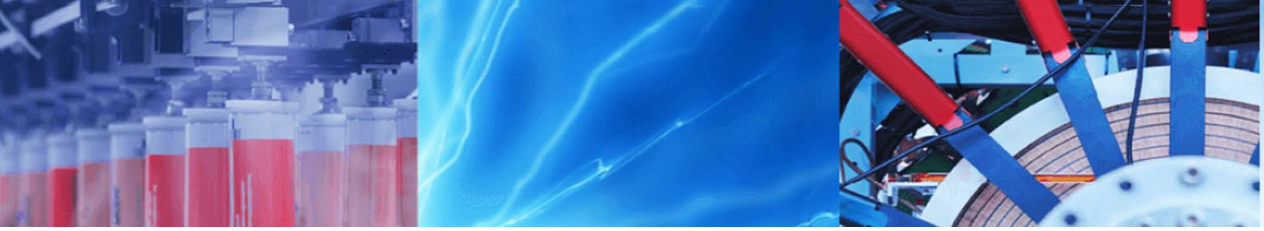

Research Article

\title{
Investigation of GaN/InGaN thin film growth on ITO substrate by thermionic vacuum arc (TVA)
}

\author{
Erman Erdoğan ${ }^{1}$ (1) $\cdot$ Mutlu Kundakçı ${ }^{2}$
}

(c) Springer Nature Switzerland AG 2018

\begin{abstract}
Group-III nitride semiconductors covering infrared, visible and ultraviolet spectral range have direct bandgaps changing from $0.7 \mathrm{eV}(\mathrm{InN})$ to $3.4 \mathrm{eV}(\mathrm{GaN})$. With this feature, optoelectronic devices such as light emitting diodes, laser diodes and ultraviolet (visible rays-UV) photodetectors are made. It is possible to grow high-quality InGaN epitaxial layers by modern crystal growth techniques such as molecular beam epitaxy, radio frequency sputtering method and metal organic chemical vapor deposition. Compared with these methods, the Thermionic Vacuum Arc, which is promising thin film growth technique, is relatively inexpensive and quite effective approach for preparing InGaN thin films. The purpose of this research is to investigate the physical properties of the film. The XRD patterns of the InGaN thin films deposited on the ITO substrate exhibited polycrystal structure. The larger crystallite size and smaller FWHM indicate better crystallization. Microstrain values also exhibit good crystallite films respect to the low dislocation density. This film has a potential for photovoltaic devices based on the absorbance graph. It was observed that the compound is homogeneously dispersed on the surface and that there is a nanoporous structure.
\end{abstract}

Keywords GaN/InGaN · Thin film growth · Thermionic vacuum arc (TVA) · Physical properties

\section{Introduction}

III-V Nitride semiconductors have many applications. Each energy bandgap (0.7-6.2 eV) between III-Nitride semiconductors forms a large series of triple alloys $[2,8,15,22]$. With this feature, optoelectronic devices such as light emitting diodes (LEDs), laser diodes and ultraviolet (visible rays-UV) photodetectors are made [12, 21, 29]. III-Nitride systems (InN, GaN, and AIN) are known as wide bandgap semiconductors. Due to their direct bandgap, they have high absorption constants and a sharper cut-off wavelength. By modifying the molar fraction of the triple alloys, the wavelength cutoff can be adjusted and the capacity of the multi-joint devices can be increased with wide bandgap engineering [18]. With all these advantages, III-Nitride structures are the most suitable materials for fabrication of optoelectronic devices in blue and ultraviolet (UV) spectral regions. It is possible to grow high-quality InGaN epitaxial layers by modern crystal growth techniques such as molecular beam epitaxy (MBE) $[7,9,10,13,19,20,23$, $30]$, radio frequency sputtering technique (RFSM) [11, $14,16,37-39]$ and metal organic chemical vapor deposition (MOCVD) $[3-5,17,26,36,40]$. The method of epitaxial crystal growth with a molecular beam involves the reaction of a thermal beam of atoms or molecules with a crystal surface in a very high vacuum environment. It is also a highly advanced single crystal growth technique in which the film thickness can be controlled very precisely. The fact that the speed of the crystal growth process is too slow is also not suitable for mass production and the

Electronic supplementary material The online version of this article (https://doi.org/10.1007/s42452-018-0013-z) contains supplementary material, which is available to authorized users.

Erman Erdoğan, e.erdogan@alparslan.edu.tr| ${ }^{1}$ Department of Physics, Faculty of Art and Science, Muş Alparslan University, Muş 49250, Turkey. ${ }^{2}$ Department of Physics, Faculty of Science, Atatürk University, Erzurum 25240, Turkey.

SN Applied Sciences (2019) 1:9| https://doi.org/10.1007/s42452-018-0013-z

Received: 3 July 2018 / Accepted: 27 September 2018 / Published online: 12 October 2018 
installation and operation of the devices are very costly [31]. Sputtering deposition is a phenomenon in which the surface atoms of a target material are dislodged by ionized gas atoms and then deposited onto the desired substrate where the ejected atoms are coated with a thin layer. Especially in sputtering technique, it is absolutely necessary to use a buffer gas. This buffer causes the formation of undesirable impurities in the produced films during the analysis of the materials covered by the gas [1]. Therefore, the Thermionic Vacuum Arc (TVA) technique has an important place in terms of impurity. The chemical vapor deposition method is to coat the surface of the heated material in an average closed vessel with a solid material resulting from a chemical reaction of a carrier gas in the vapor state. However, the reactive gases used in the coating are usually dangerous and expensive gases and some unintended components that form as a result of the reaction can affect the coating base [27]. Compared with these methods TVA, which is promising thin film growth technique, is relatively inexpensive and quite effective approach for preparing InGaN thin films. In addition, this method is good for depositing films with high crystallinity and is advantageous for the preparation of films with a short production time. With TVA technique it is possible to work in a high vacuum or very high vacuum conditions. Due to these vacuum conditions, the quality of coated film increases and the effects that may occur on the coated film due to oxidation or some gases are minimized [41].

In this research, InGaN thin films have been deposited on GaN and indium tin oxide (ITO) substrate by TVA. The purpose of this research is to investigate some physical properties of InGaN thin films. XRD was used to analyze the crystal structure of the deposited films. The optical properties of the films have been studied via absorption measurements with ultraviolet-visible spectroscopy (UV/ VIS) in the wavelength $300-800 \mathrm{~nm}$. Surface morphology properties of the glass/GaN/InGaN film structure were investigated by an Atomic Force Microscopy (AFM). The structure and surface morphology of deposited InGaN thin film was determined by scanning electron microscopy (SEM). Compositional analysis was done by energy dispersive $\mathrm{X}$-ray spectroscopy (EDAX).

\section{Experimental}

GaN and InGaN films on ITO substrate were grown by TVA with very short production time being $40 \mathrm{~s}$ for $\mathrm{GaN}$ and $90 \mathrm{~s}$ for InGaN. The film was deposited at $2 \times 10^{-4} \mathrm{Torr}$ working pressure, $18.5 \mathrm{~A}$ filament current. Plasma was produced at $200 \mathrm{~V}$ for $\mathrm{GaN}$ and $500 \mathrm{~V}$ for $\ln \mathrm{GaN}$, at $0.5 \mathrm{~A}$ plasma current. ITO has been preferred as a substrate material over types of the metal oxides. Because ITO is a well-known transparent semiconducting oxide thin film. Transparent and conductive layers on substrates are an important component of today's optoelectronic technology. ITO substrate have been bought from Sigma-Aldrich Co. With $70-100$ ohms/sq surface resistivity. TVA is a new and different technique from other techniques that produce plasma in anode metal vapors for the regulation of electrodes. The TVA takes place among the anode where the material is placed and a thermionic cathode which is heated directly $[24,28,34]$. Since there is no high temperature during coating process, there is no thermal expansion problem in the coating of metals [25]. Structure of the thin films was studied by XRD with Empyrean, PANalytical with $\mathrm{Cu} \mathrm{K}_{\mathrm{a}}$ radiation. The measurements were performed at grazing incidence angle of $0.5^{\circ}$. One of the most commonly used methods for determining absorption coefficient and energy bandgap of semiconductors is the absorption measurement method. Absorbance measurements were taken using a double-beam UV-vis Spectrometer (300-800 nm) with a Shimadzu UV-3600 Plus model. SEM images and EDX spectrum measurements were taken with a Sigma 300 Model Zeiss Gemini FEG-SEM device. The morphologies of the produced thin films were obtained with Hitachi AFM 5000 II Model device using AFM (dynamic force mode measurement) device. All characterization measurements have been taken at East Anatolian High Technology Application and Research Center (DAYTAM).

\section{Results and discussion}

The thickness of deposited InGaN film was measured using an interferometer and the thickness measurement system Filmetrics F20. The average thickness of the thin film was found to be about $250 \mathrm{~nm}$. XRD in the $2 \theta$ range of $10^{\circ}-80^{\circ}$ was used for crystallographic data of InGaN thin film deposited on ITO substrate at room temperature. X-ray diffraction patterns of deposited thin films are shown in Fig. 1.

The XRD patterns of the InGaN thin films deposited on the ITO substrate exhibited polycrystal structure with three diffraction peaks at $30.30^{\circ}$ corresponding to the (100) plane of the $\operatorname{lnGaN}, 33.00^{\circ}$ and $36.30^{\circ}$ corresponding to the (0002) and (10-11) planes of the GaN, respectively. Wang et al. [39] found (002), (100) and (101) peaks of $\ln _{x} G a_{1-x} N$ films deposited on silicon for different In compositions. They reported that deposited films have hexagonal crystal system. (The obtained results for parameters are given in the supplementary material file). For ITO substrate $50.60^{\circ}$ and $54.50^{\circ}$ are observed with d values of $1.81 \AA$ and $1.69 \AA$, respectively. 


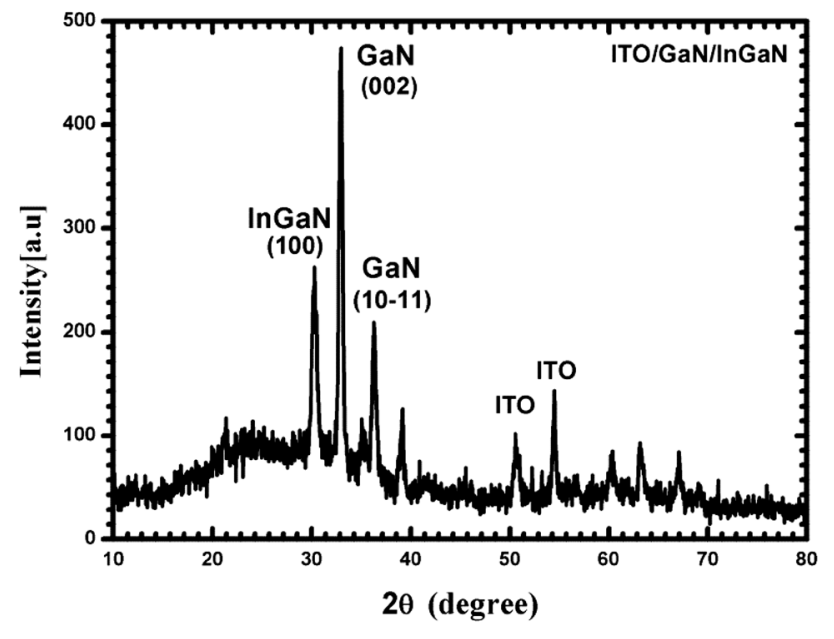

Fig. 1 XRD diffraction patterns of InGaN thin films grown on ITO substrate by TVA method

Some specific properties of the grown films are determined by utilizing the XRD pattern. The particle size of the deposited films was calculated using the Debye-Scherrer formula given at;

$L=\frac{K \lambda}{\beta \cos \theta}$

where $\mathrm{K}$ is shape constant, which is $0.94, \lambda$ is the $\mathrm{X}$-ray wavelength, $\theta$ is the Bragg diffraction angle, and $\beta$ is the full width at half-maximum (FWHM) of the XRD peak appearing at the diffraction angle. In crystals, point defects such as the vacant atomic sites in a structure or the extra atoms positioned between atomic sites may occur. This leads to a change in the conductivities, transmissivities and many properties of the films. Dislocations are linear defects in the crystal structure. Dislocations play an important role in the growth mechanism of films. Dislocation density $(\delta)$ is defined as the dislocation line length in the unit volume and is a measure of the number of defects in the crystal. The dislocation density of the films is calculated by the following equality:

$\delta=\frac{1}{L^{2}}$

We used the following expression to determine the microstrain:

$\epsilon=\frac{\beta \cos \theta}{4}$

where $\beta$ is the width at half-maximum (FWHM) and $\theta$ is the diffraction Bragg angle. Additionally, crystal number (N) of produced InGaN thin films calculated using the following equation:
$N=\frac{t}{L^{3}}$

where $t$ is the thickness of the produced film. (The obtained results for parameters are given in the supplementary material file). It is seen that the predicted dislocation density and microstrain values are inversely proportional to the crystallite size values. The larger crystallite size, smaller FWHM and indicate better crystallization. Microstrain values also exhibit good crystallite films respect to the low dislocation density. Similar studies have been reported for wide band gap semiconductor material $\mathrm{ZnO}$ with different doping situations. Highest peak value of XRD, the narrowest value of FWHM, large crystallite size and low dislocation density are the major parameters that show the better crystal structures [32, 35].

One of the most commonly used methods for determining optical absorption and energy band range of semiconductors is the absorption measurement method. Absorption measurements of thin films on the ITO substrate were taken at room temperature with the aid of a Shimadzu UV-3600 Plus spectrometer. The spectrometric measurement range used is $300-800 \mathrm{~nm}$. In the measurements made, information about the absorption and bandgap of the material was obtained. As can be seen in Fig. 2a, below $450 \mathrm{~nm}$ there is a strong absorbance in the film. On the other hand, absorbance values are almost same, after the value of $450 \mathrm{~nm}$, which results in low absorption losses in the visible range. This film indicates a good optical quality and has a potential for photovoltaic devices based on the absorbance graph.

The absorption of light by an optical medium is quantified by its absorption coefficient (a), which defined as the fraction of the power absorbed in a unit length of the medium given by

$\alpha=2.303 \frac{A b s}{t}$

where $t$ is the thickness of the produced film. In Fig. 2b, the value of the optical band gap is calculated as $2.53 \mathrm{eV}$ with the fit plot drawn on the energy graph of $(\mathrm{ahu})^{2}$ $\left(\mathrm{cm}^{-1} \mathrm{eV}\right)^{2}$ of the GaN/InGaN thin film grown on the ITO by RFMS method. This result also supports absorbance values against wavelength. The value of optical band gap of ITO susbtrate is calculated as $4.14 \mathrm{eV}$, which is shown in Fig. $2 \mathrm{~b}$. The optical band gap for ITO as a thin film was found to be in the range of 4.01-4.03 eV [6].

It is very important to investigate surface morphology of the films. For studying surface morphology, Sigma 300 Model Zeiss Gemini FEG-SEM device was used. SEM images are shown in Fig. 3. In Fig. 3, the GaN/InGaN compound grown at $1 \mu \mathrm{m}$ and $200 \mathrm{~nm}$ magnification at $3.8 \mathrm{~mm}$ working distance with the in-lens detector. It was observed that 

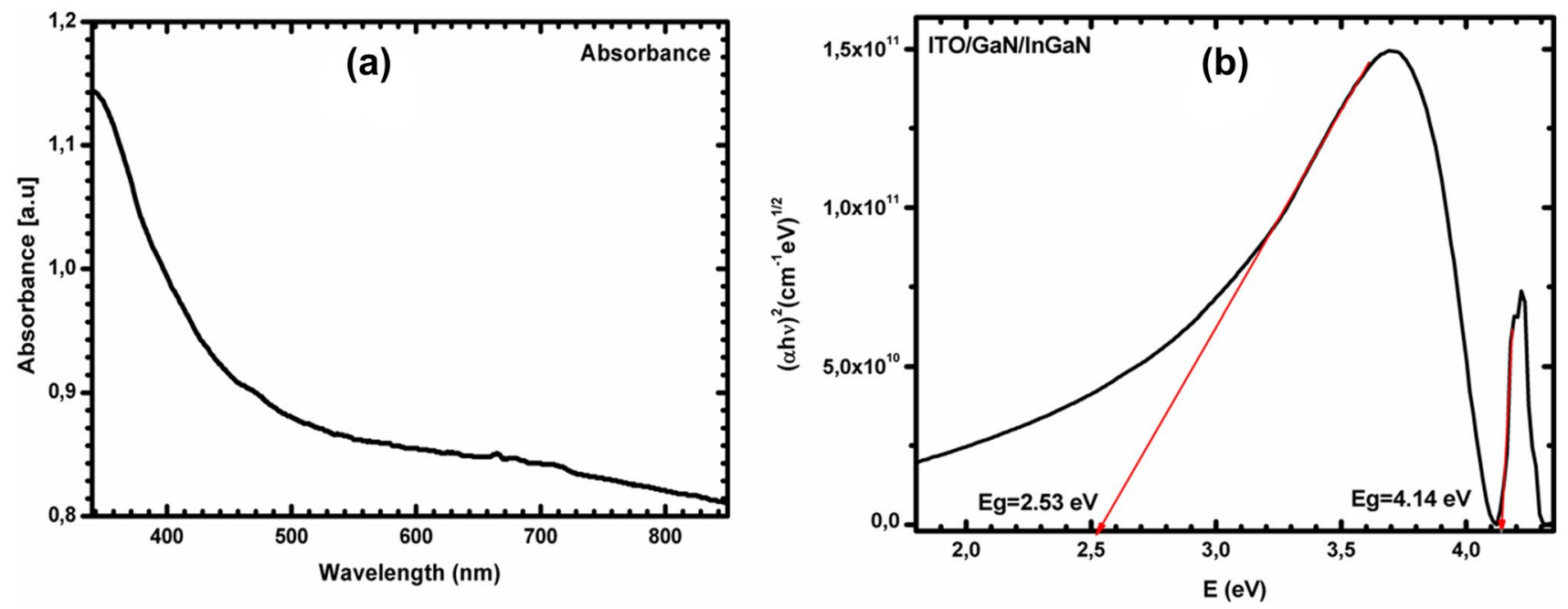

Fig. 2 a Absorption graph against wavelength and $\mathbf{b}(\mathrm{ahu})^{2}\left(\mathrm{~cm}^{-1} \mathrm{eV}\right)^{2}$ graph against energy of GaN/InGaN thin film
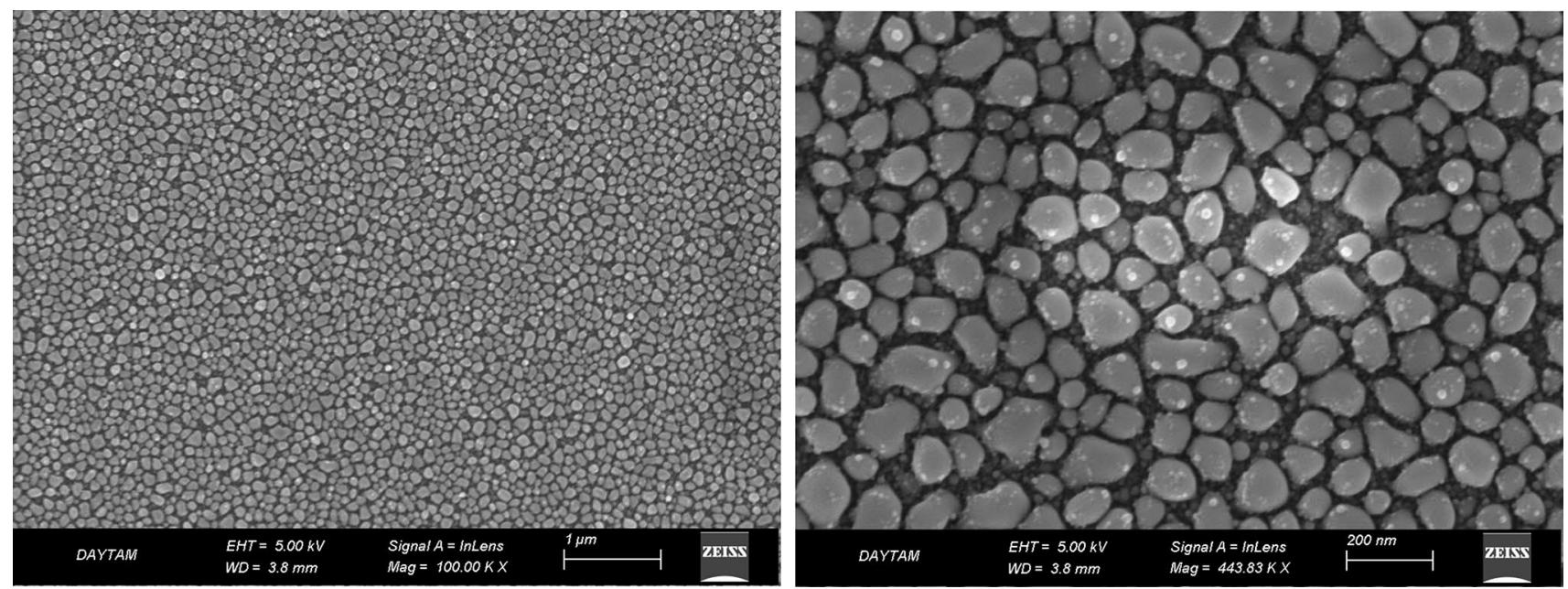

Fig. 3 Different magnitudes of FEG-SEM images of GaN/InGaN thin film grown on ITO by TVA method

the compound is homogeneously dispersed on the surface and that there is a nanoporous structure. In addition, as seen in the image, thin film has a granular structure. The deposited film is dense, smooth, and continue without any holes and cracks.

We see that the EDX results reveal that nitrogen, oxygen, gallium, silicon and indium are the main elements present within the inspection field, with oxygen being the most abundant. The tabulated results provide a semiquantitative view of the elemental composition in the inspection field in units of both weight percent and atomic percent. The elemental analysis results showed that In composition is $12.37 \%$ in the InGaN alloy. (EDX spectrum and elemental analysis results are given in the supplementary material file).
The $5 \mu \mathrm{m} \times 5 \mu \mathrm{m}$ scale atomic force microscope (AFM) images of the top surface were performed in dynamic force mode by using Hitachi AFM 5000 II Model device using AFM device. Surface morphology images of the film are showed in Fig. 4. The linear roughness value is $6.86 \mathrm{~nm}$ and the average roughness value is $5.41 \mathrm{~nm}$, which is consistent with each other. Height of the highest peak above the mean line in the profile (Rp) was $23.23 \mathrm{~nm}$ and depth of the deepest valley below the mean line in the profile (Rv) was $13.09 \mathrm{~nm}$. Hence, total roughness (Rt), which is the sum of the (Rp) and (Rv) and was calculated as $36.32 \mathrm{~nm}$. (The obtained results for parameters are given in the supplementary material file). Saleh et al. [33] synthesized $\mathrm{In}_{0.08} \mathrm{Ga}_{0.92} \mathrm{~N} / \mathrm{AIN} / \mathrm{Si}$ thin films with a photoelectrochemical etching technique. They have investigated the 


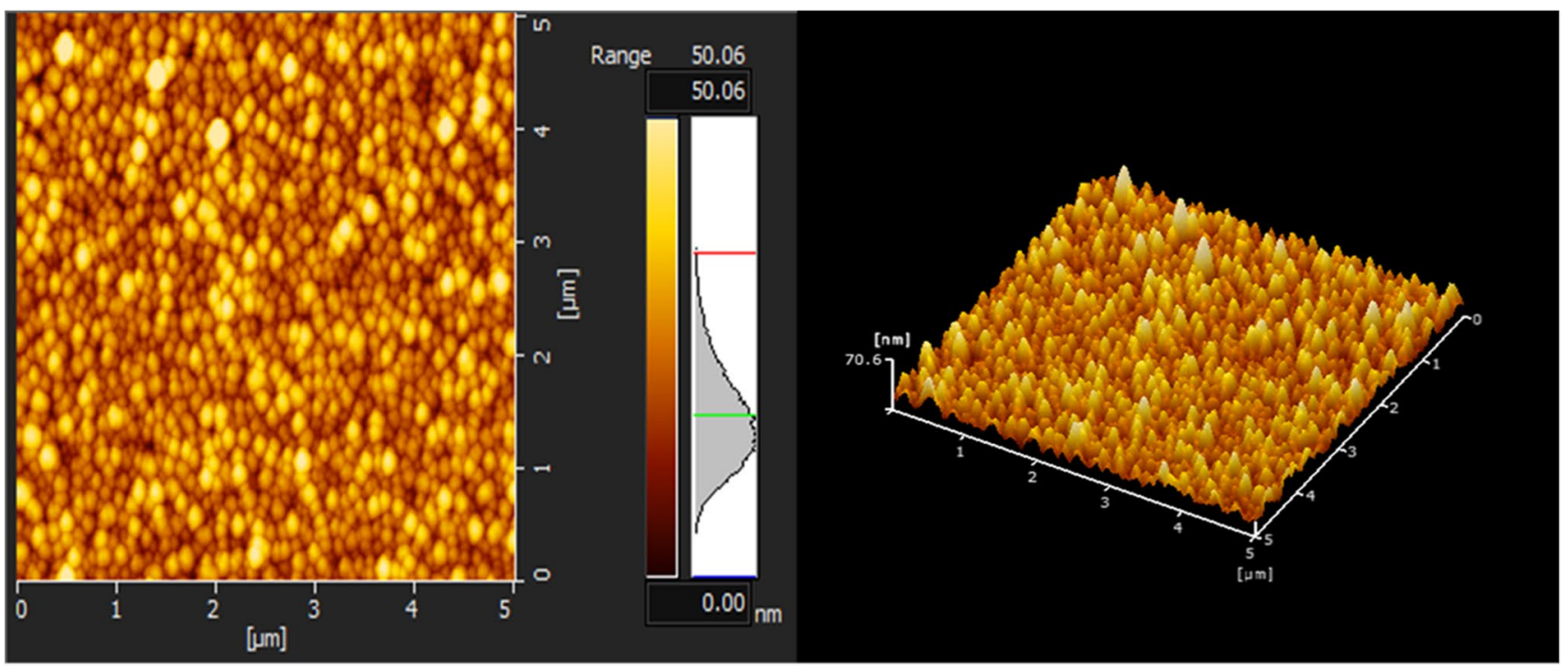

Fig. 4 The 2D and 3D AFM images of the produced film

effects of etching durations on films morphology. Root mean square (RMS) roughness of that film was found to be $2.2 \mathrm{~nm}$.

\section{Conclusion}

GaN/InGaN thin film was deposited on ITO substrate to analyze its physical properties. We successfully achieved hexagonal GaN/InGaN thin film on ITO substrate using TVA method. The XRD patterns of the InGaN thin films deposited on the ITO substrate exhibited polycrystal structure with three diffraction peaks at $30.30^{\circ}, 33.00^{\circ}$ and $36.30^{\circ}$ corresponding to the (100), (0002) and (10-11) planes of the InGaN, respectively. Below $450 \mathrm{~nm}$ there is a strong absorbance in the film. On the other hand, absorbance values are almost same, after the value of $450 \mathrm{~nm}$, which results in low absorption losses in the visible range. The value of the energy bandgap is calculated as $2.53 \mathrm{eV}$ with the fit plot drawn on the energy graph of (ahu $)^{2}$ $\left(\mathrm{cm}^{-1} \mathrm{eV}\right)^{2}$ of the GaN/InGaN thin film grown on the ITO by RFMS method. This result also supports absorbance values against wavelength. SEM images are shown that the films are crystalline with a uniform dimension of crystals. Surface morphology images of the film indicated that the linear roughness value is $6.86 \mathrm{~nm}$ and the average roughness value is $5.41 \mathrm{~nm}$, which is consistent with each other. Elemental analysis results confirmed the presence of the $\mathrm{Si}, \mathrm{Ga}, \mathrm{In}, \mathrm{N}$ and $\mathrm{O}$ in the film. Finally, TVA is a fast growth method for GaN/InGaN thin film.

Acknowledgement We are thankful to Prof. Suat Pat and Dr. Volkan Şenay (Dept. of Physics, Osmangazi University, Eskişehir), for sharing their lab to use TVA growth method and we would like to thank Dr. Emre Gür (Physics Dept., Atatürk University,) for all characterization measurements made at DAYTAM.

\section{Compliance with ethical standards}

Conflict of interest The authors declare that they have no conflict of interest.

\section{References}

1. Alfonso E, Jairo O, Gloria C (2012) Thin film growth through sputtering technique and its applications. Crystallization-Science and Technology, InTech

2. Ambacher O (1998) Growth and applications of group IIInitrides. J Phys D Appl Phys 31(20):2653

3. Chang PC, Yu CL, Jahn YW, Chang SJ, Lee KH (2011) Effect of growth temperature on the indium incorporation in InGaN epitaxial films. Adv Mater Res 287:1456

4. Chen PC, Chen CH, Chang SJ, Su YK, Chang PC, Huang BR (2006) High hole concentration of $\mathrm{p}$-type InGaN epitaxial layers grown by MOCVD. Thin Solid Film 498(1-2):113

5. Chen YS, Liao CH, Kuo CT, Tsiang RCC, Wang HC (2014) Indium droplet formation in InGaN thin films with single and double heterojunctions prepared by MOCVD. Nanos Res Lett 9(1):334

6. Du J, Chen XL, Liu CC, Ni J, Hou GF, Zhao Y, Zhang XD (2014) Highly transparent and evaporation at low temperature. Appl Phys A 117(2):815

7. Fabien CA, Gunning BP, Doolittle WA, Fischer AM, Wei YO, Xie H, Ponce FA (2015) Low-temperature growth of InGaN films over the entire composition range by MBE. J Crys Growth 425:115

8. Feng ZC (ed.) (2008) III-nitride devices and nanoengineering. World Scientific

9. Gherasoiu I, Yu KM, Reichertz LA, Kao VM, Hawkridge M, Ager JW, Walukiewicz W (2010) High quality InxGa1-xN thin films with $x>0.2$ grown on silicon. Phys Status Solidi (b) 247(7):1747

10. Gherasoiu I, Yu KM, Reichertz L, Walukiewicz W (2015) InGaN pn-junctions grown by PA-MBE: material characterization and 
fabrication of nanocolumn electroluminescent devices. J Crys Growth 425:393

11. Guo Q, Kusunoki Y, Ding Y, Tanaka T, Nishio M (2010) Properties of InGaN films grown by reactive sputtering. Japan J Appl Phys 49(8R):081203

12. Huang CF, Hsieh WY, Hsieh BC, Hsieh CH, Lin CF (2013) Characterization of InGaN-based photovoltaic devices by varying the indium contents. Thin Solid Film 529:278

13. Islam SM, Protasenko V, Rouvimov S, Xing HG, Jena D (2016) Sub-230 nm deep-UV emission from GaN quantum disks in AIN grown by a modified Stranski-Krastanov mode. Japan J Appl Phys 55(5S):05FF06

14. Itoh T, Hibino S, Sahashi T, Kato Y, Koiso S, Ohashi F, Nonomura $S$ (2012) InXGa1-XN films deposited by reactive RF-sputtering. J Non-Crys Solids 358(17):2362

15. Jain SC, Willander M, Narayan J, Overstraeten RV (2000) IIInitrides: growth, characterization, and properties. J Appl Phys 87(3):965

16. Jakkala P, Kordesch ME (2017) Bandgap tuning and spectroscopy analysis of $\operatorname{In} x \mathrm{Ga}(1-\mathrm{x}) \mathrm{N}$ thin films grown by RF sputtering method. Mater Res Exp 4(1):016406

17. Kadys A, Malinauskas T, Grinys T, Dmukauskas M et al (2015) J Electron Mater 44(1):188

18. Kern R, Scott CC, Goetz W, Kuo C (2001) U.S. Patent No. 6, 194, 742

19. Lieten RR, Tseng WJ, Yu KM, Van de Graaf W, Locquet JP, Dekoster J, Borghs G (2013) Single crystalline In x Ga 1-x N layers on germanium by molecular beam epitaxy. Cryst Eng Commun 15(44):9121

20. Ma QB, Lieten R, Degroote S, Germain M, Borghs G (2015) Indium-rich InGaN films grown on Ge substrate by plasmaassisted molecular beam epitaxy for solar water splitting. J Electron Mater 44(1):202

21. Morkoç H (2009) Handbook of nitride semiconductors and devices, materials properties, physics and growth. Wiley

22. Morkoç H, Hull R, Osgood Jr. RM, Sakaki H, Zunger A (1999) General properties of nitrides. Springer, Berlin, pp 1-39, 45-80, 83-141, 163, 332-336

23. Nanishi Y, Saito Y, Yamaguchi T, Hori M et al (2003) MBE-growth, characterization and properties of $\ln N$ and $\ln G a N$. Phys Status Solidi (a) 200(1):202

24. Özen S, Şenay V, Pat S, Korkmaz Ş (2016) The influence of voltage applied between the electrodes on optical and morphological properties of the InGaN thin films grown by thermionic vacuum arc. Scanning 38(1):14

25. Özen S, Pat S, Şenay V, Korkmaz Ş (2017) The surface morphology research of the $\mathrm{BGaN}$ thin films deposited by thermionic vacuum arc. Vacuum 135:50
26. Pantha BN, Dahal R, Li J, Lin JY, Jiang HX, Pomrenke G (2008) Thermoelectric properties of In x Ga $1-x \mathrm{~N}$ alloys. Appl Phys Lett 92(4):042112

27. Park JH, Sudarshan TS (Eds.) (2001) Chemical vapor deposition, (Vol. 2) ASM international

28. Pat S, Korkmaz Ş, Özen S, Şenay V (2015) GaN thin film deposition on glass and PET substrates by thermionic vacuum arc (TVA). Mater Chem Phys 159:1

29. Patrick $M$ (2010) LED for lighting applications. Wiley

30. Reichertz LA, Yu KM, Cui Y, Hawkridge ME et al. (2008) InGaN thin films grown by ENABLE and MBE techniques on silicon substrates. In: MRS Online Proceedings Library Archive, p 1068

31. Rinaldi F (2002) Basics of molecular beam epitaxy (MBE). UNIVERSITÄT ULM, 31

32. Salaken SM, Farzana E, Podder J (2013) Effect of Fe-doping on the structural and optical properties of $\mathrm{ZnO}$ thin films prepared by spray pyrolysis. J Semicon 34(7):073003

33. Saleh HA, Hassan Z, Yam FK (2015) Int J Nanoelectron Mater 8:33

34. Şenay V, Özen S, Pat S, Korkmaz Ş (2016) Optical, structural, morphological and compositional characterization of a Co-doped GaAs semiconducting thin film produced by thermionic vacuum arc. J Alloys Comp 663:829

35. Tahir D, Jae KH (2017) In: AIP Conference Proceedings, AIP Publishing. 1801(1): 020007

36. Tao T, Zhao Z, Lian L, Hui S et al (2011) Surface morphology and composition studies in InGaN/GaN film grown by MOCVD. J Semicon 32(8):083002

37. Wang J, Shi XJ, Zhu J (2013) Low-temperature growth of InxGa1 - xN films by radio-frequency magnetron sputtering. Appl Surf Sci 265:399

38. Wang J, Hou XH, Shi XJ, Zhu J (2015) Effects of nitrogen flow ratio on the properties of radiofrequency-sputtered InGaN films. J Electron Mater 44(4):1160

39. Wang X, He L, Li X, Su X, Zhang Z, Zhao W (2016) Structural and morphological characteristics of Inxga1 - xN films grown on SI (111) by reactive magnetron sputtering. MATEC Web Conf EDP Sci 67:04013

40. Yang PF, Jian SR, Lai YS, Yang CS, Chen RS (2008) Morphological, structural, and mechanical characterizations of InGaN thin films deposited by MOCVD. J Alloys Comp 463(1-2):533

41. Yudar HH, Pat S, Korkmaz Ş, Özen S, Şenay V (2017) Zn/ZnSe thin films deposition by RF magnetron sputtering. J Mater Sci Mater Electron 28(3):2833 